\title{
Trends in Optical Span Loss Detected Using the Time Series Decomposition Method
}

\author{
Banti Laure M. Yaméogo, Douglas W. Charlton, David Doucet, Christian Desrosiers, \\ Maurice O'Sullivan and Christine Tremblay ${ }^{1}$
}

\begin{abstract}
Optical fiber cables deployed in the last 30 years have been considered to be long-lived components due to the generally high level of reliability of glass and the robustness of cable construction. Like all other components however, optical cables can change with age as a result of their construction or deployment characteristics as well as operational and environmental stress factors. In this paper, we apply a time-series decomposition method on span loss data collected during 12 months in four bidirectional spans of a production network in order to detect long-term degradation of the fiber plant. After extracting the trend component, a Mann-Kendall test is applied to the span loss curves and a Sen's slope estimator test is used for determining the magnitude of span loss change. The method allows detecting a $1.3 \%$ increase in span loss over the 12 -month period of observation in one of the fiber spans. This trend is confirmed using a linear regression model computed according to Theil Sen method applied to additional one-year data. The proposed method can allow the early detection of fiber plant degradation and the proactive planning of fiber replacement.
\end{abstract}

Index Terms - Linear regression, Long term trend detection, Mann-Kendall, Optical Erbium-doped fiber amplifiers, Optical fiber communication, Optical fiber losses, Sen's slope, STL, Time series decomposition method.

\section{INTRODUCTION}

$\mathrm{T}$ HE optical fiber cables in long haul, metropolitan and access networks are composed of fiber spans of different lengths and numbers, the optical span loss must always be managed carefully for error-free data transmission [1]. Several generations of optical fiber cables exist in today's networks. The quality of transmission of the optical signals carried in fiber links changes over time as a result of linear and nonlinear channel interactions, maintenance operations in the outside plant and environmental factors, as well as long-term component drift and aging effects [2].

${ }^{1}$ Manuscript submitted on February 17, 2020.

This work was supported by the Natural Sciences and Engineering Research Council (NSERC) of Canada under grant CRDPJ 488332-15.

B. L. M. Yaméogo, C. Tremblay are with the Network Technology Lab, Department of Electrical Engineering, École de technologie supérieure, Montréal, Qc, Canada H3C 1K3 (e-mail: christine.tremblay@etsmtl.ca).

C. Desrosiers is with Laboratory for Imagery Vision and Artificial Intelligence, Department of Software and IT Engineering, École de technologie supérieure, Montréal, Qc, Canada H3C 1K3.

D. W. Charlton, D. Doucet and M. O'Sullivan are with Ciena Corp., Ottawa, ON, Canada K2K 0L1
Network operators monitor the optical performance of their networks on a continuous basis for reliable data transmission. Legacy network control systems can allow real-time monitoring of system and link parameters such as the optical power levels at the terminal and amplifier sites, as well as the bit error rate (BER) at the receiver. These performance parameters, stored in databases are used for root cause analysis in case of component or link failure. As fiber plant becomes older, network operators seek to quantify long-term fiber plant aging effects.

Characterizing the long-term performance of optical fiber cables deployed for more than 30 years is still an open problem. Much work has been devoted to this problem so far, but the optical fiber aging process is not fully understood [3]. Various theoretical models have been applied for mechanical characterization of optical fibers. The most common one is based on Weibull's statistics. The Weibull law expresses the failure probability of an optical fiber subjected to an applied stress [4]. Other physical techniques have been developed, such as the measurement of the tensile strength or elongation-at-break of the insulation material; however, these methods are inherently destructive and require a specimen of the aged cable for testing [5]. A cable and method for monitoring its ageing has been proposed, but this approach cannot be applied to legacy fiber plants [6]. More recently, optical time-domain reflectometer (OTDR) and power measurements performed on a 24-fiber optical fiber cable 16 years after deployment on a $34-\mathrm{km}$ route showed an average attenuation loss increase of $20 \%$ which was attributed to the presence of water in protective fiber optic closure in this specific case [7].

In this paper, we propose a time series decomposition method for detecting long-term trends in fiber span loss. We test the method with field data collected in a 4-span bidirectional optical link of a production network, using three complementary statistical approaches widely used in the domain of meteorology, finance, and air/water quality monitoring: locally weighted regression (Loess) smoothing (STL), Mann-Kendall, and Sen's slope tests [8].The remainder of the document is organized as follows. In section II, the time series data and the decomposition method are described. In addition, statistical tests and the linear regression method are also covered. The results are presented in section III. Conclusions are drawn in section IV. 


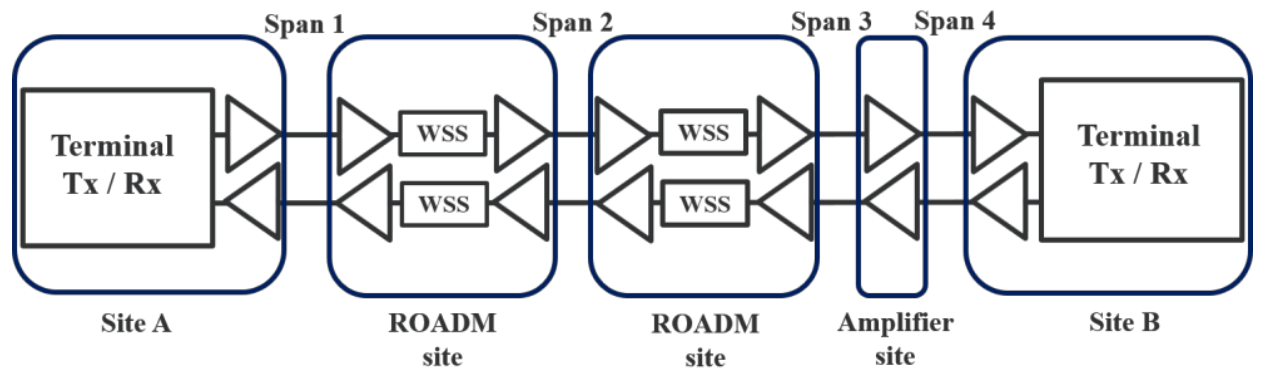

Fig. 1. Bidirectional optical link under analysis (4 fiber spans, $205 \mathrm{~km}$ total length). WSS: wavelength selective switch; ROADM: Reconfigurable Optical Add-Drop Multiplexer.

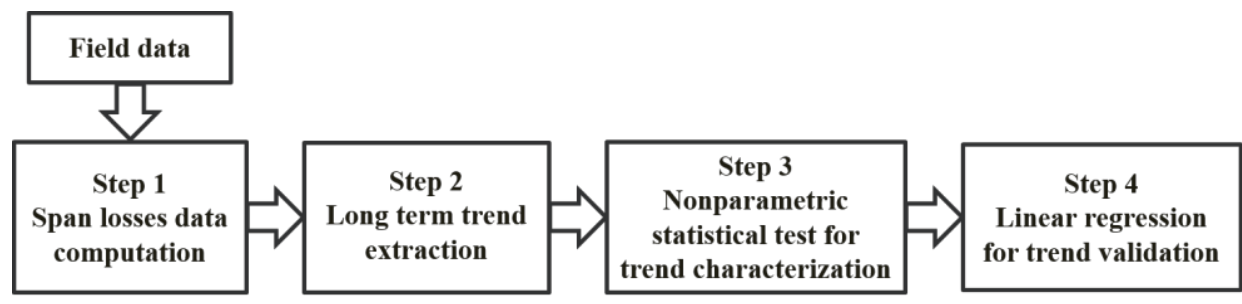

Fig. 2. Methodology

TABLE I

STATISTICAL ANALYSIS OF THE SPAN LOSS DATA

\begin{tabular}{|c|c|c|c|c|c|c|c|}
\hline & \multirow[b]{2}{*}{ Span } & Median & Min & Max & $\mathrm{IQR}$ & \multirow{2}{*}{$\begin{array}{l}\mathrm{CV} \\
(\%)\end{array}$} & \multirow{2}{*}{$\begin{array}{l}\gamma_{1} \\
-\end{array}$} \\
\hline & & \multicolumn{4}{|c|}{$(\mathrm{dB})$} & & \\
\hline & 1 & 13.5 & 13.3 & 13.6 & 0.1 & 0.5 & -0.6 \\
\hline Direction & 2 & 20.0 & 19.9 & 20.6 & 0.1 & 0.7 & 2.6 \\
\hline \multirow[t]{3}{*}{ A-B } & 3 & 13.1 & 13.0 & 13.2 & 0 & 0.3 & -0.3 \\
\hline & 4 & 21.1 & 21 & 21.1 & 0 & 0.1 & -0.8 \\
\hline & 1 & 13.7 & 13.6 & 13.8 & 0.1 & 0.3 & -0.5 \\
\hline Direction & 2 & 20.3 & 20.1 & 20.7 & 0.1 & 0.5 & 2.7 \\
\hline \multirow[t]{2}{*}{ B-A } & 3 & 14.2 & 14.1 & 14.2 & 0.1 & 0.2 & -0.7 \\
\hline & 4 & 18.6 & 18.5 & 18.7 & 0 & 0.1 & -1.5 \\
\hline
\end{tabular}

\section{Methodology}

\section{A. System setup and data pre-processing}

Performance monitoring (PM) datasets used in this study were collected at 15 -minute sampling intervals over 12 months on a polarization multiplexed quadrature phase shift keying (PM-QPSK) channel at $100 \mathrm{~Gb} / \mathrm{s}$ deployed in a $205-\mathrm{km}$ optically amplified optical link of a production network carrying live traffic, as shown in Fig. 1.

Fig. 2 shows the methodology used for conducting the study. Initially, the time series are constituted of span loss data calculated from the measured input and output power levels of the Erbium-Doped Fiber Amplifiers (EDFAs). Daily averages are made as the span loss does not vary much within a day. The resulting dataset contains 365 samples and only 10 missing data samples.

The second step of the methodology is to extract the long term trend by performing a decomposition of the time series into seasonal, trend, and noise components. In the third step, non-parametric Mann-Kendall and Sen's slope tests are applied for characterizing the trend component. A linear regression is applied for trend validation in the last step.

Before performing the different analyses, it is important to have a profile of the data. Therefore, an overview of the basic statistical characteristics of the span loss data is shown in TABLE I. For the purpose of the analysis, span 1 and 3, and span 2 and 4, were grouped as they exhibit comparable median span loss: around $13 \mathrm{~dB}$ for spans 1 and 3 , around $20 \mathrm{~dB}$ for spans 2 and 4. No significant variation in span loss was observed during the 12-month period, as expected and confirmed by coefficients of variation $(\mathrm{CV})$ below $1 \%$. The loss histograms in Fig. 3-5 are relatively narrow, with an interquartile range (IQR) lower than $0.2 \mathrm{~dB}$. To test whether the different span losses data follow a normal distribution, the skewness $\left(\gamma_{1}\right)$ is computed. It is a measure of symmetry or, more precisely, the lack of symmetry in a distribution. The skewness for normal distribution is zero. Spans 1, 3, and 4, are skewed to the left and span 2 is skewed to the right. The bi-modal nature of data distributions are not due to seasonal effects, but rather to abrupt changes increase or decrease in span loss during the period of observation. The magnitude of these changes are of the order of a few tenths of a dB and likely result from maintenance actions (such as fiber re-splicing) on the fiber plant. As the data do not follow a normal distribution, non-parametric statistical test methods have been used in the following steps $[9,10]$.

\section{$B$. Time series decomposition method}

The second step of the analysis is to use the time series decomposition method to perform long-term trend detection. This method separates a series into three underlying components which combine to produce the data under investigation: a long-term progression, seasonality (seasonal variation) and a noise (remainder) term. The basic structural time series model is [11] : 

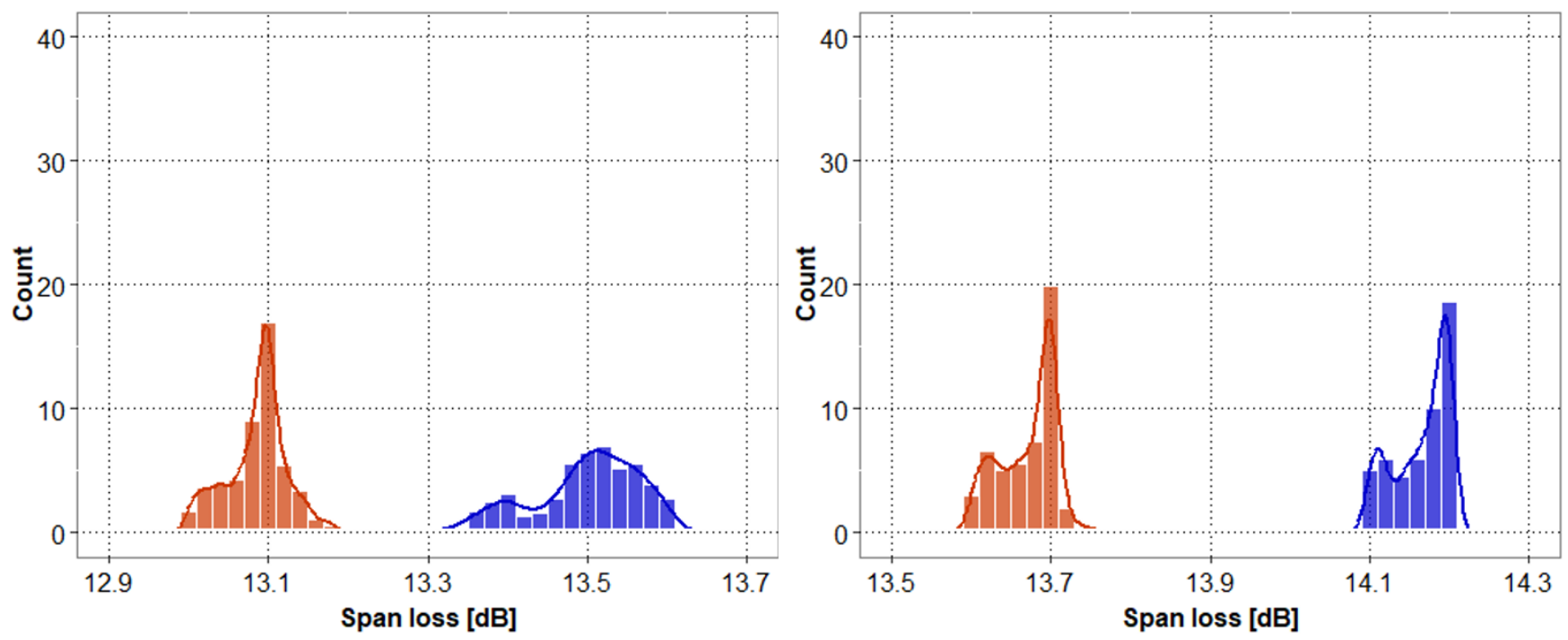

Fig. 3. Histograms for span 1 (blue), span 3 (red); Direction A - B (left), Direction B - A: (right)
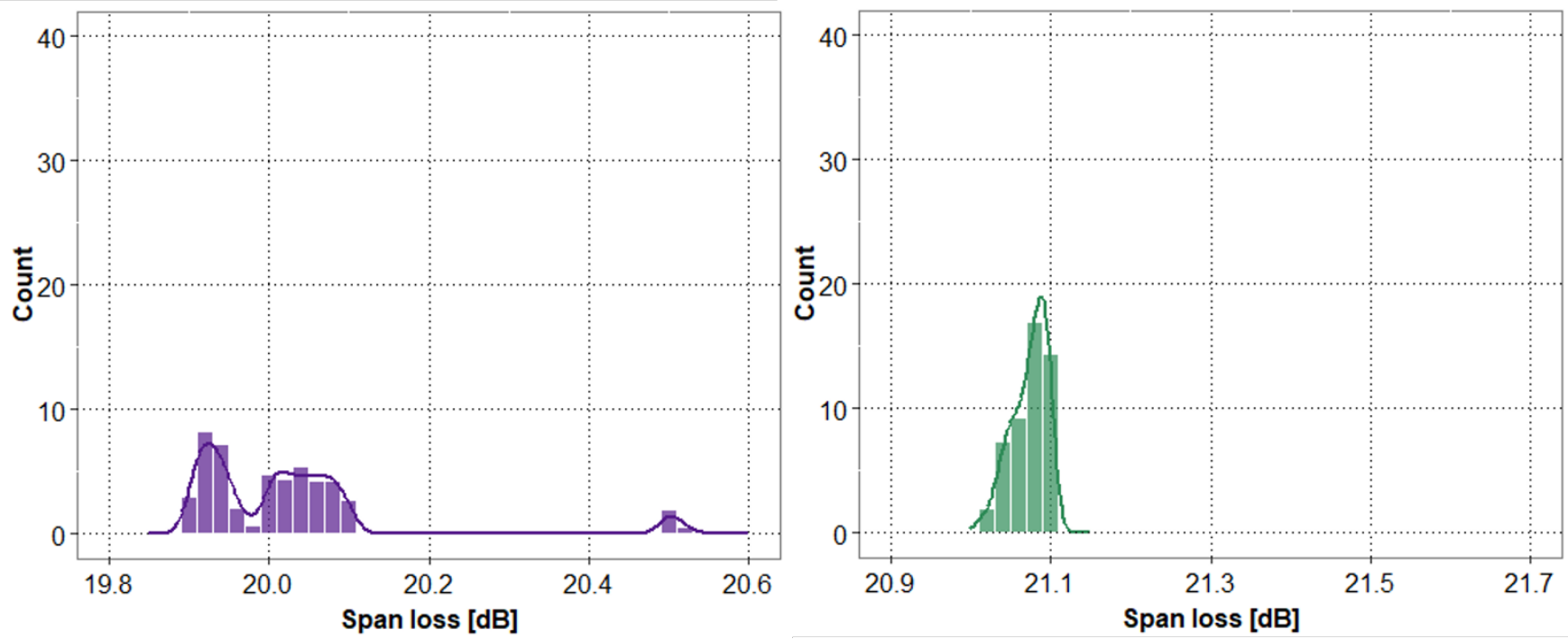

Fig. 4. Histograms for span 2 (purple), span 4 (green): Direction A - B
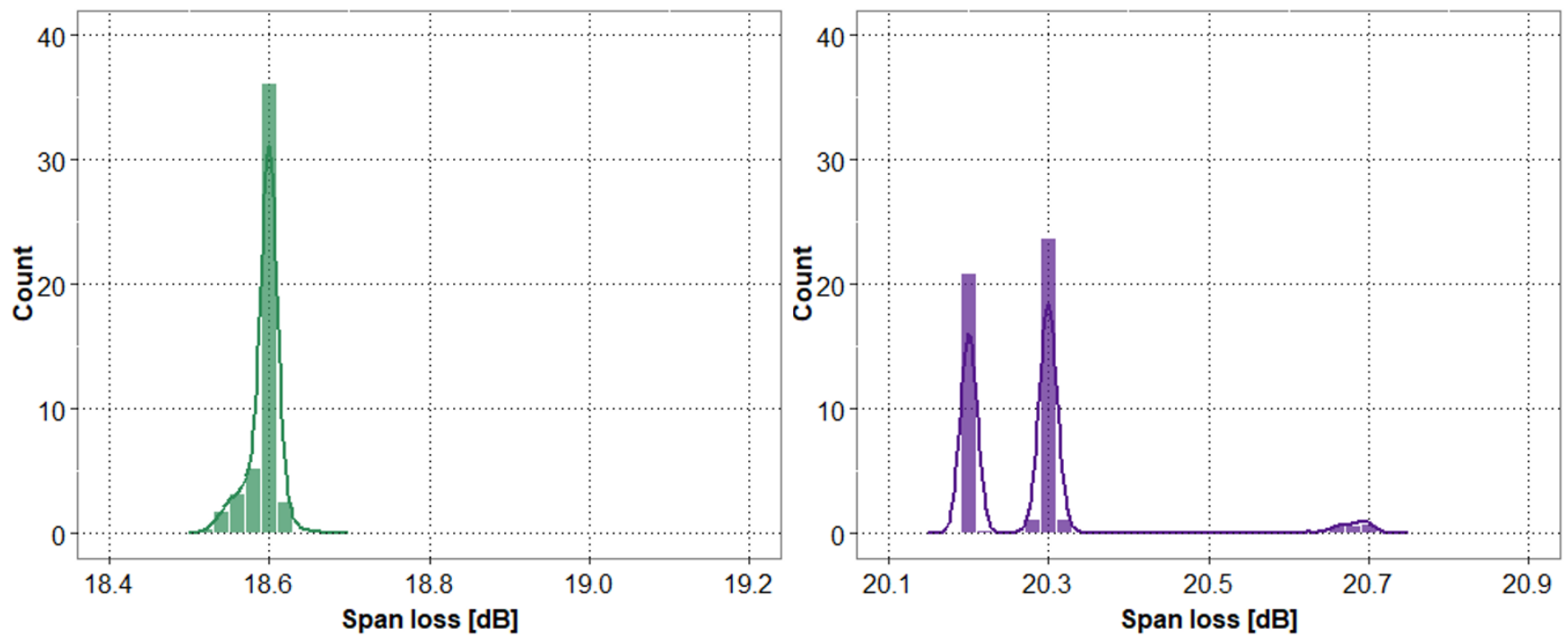

Fig. 5. Histograms for span 2 (purple), span 4 (green) : Direction B - A 


$$
Y_{t}=U_{t}+S_{t}+I_{t}, \quad \mathrm{t}=1, \ldots \mathrm{T}
$$

Where:

- $U_{t}$ is the trend component at time step t which reflects the long-term progression of the time series.

- $S_{t}$ is the seasonal component at time step $\mathrm{t}$ reflecting seasonality and

- $I_{t}$ is the irregular component (or "noise") at time step t, which represents the residuals or remainder of the time series after the other components have been removed.

It theoretically follows a normal distribution with zero mean and variance $\sigma_{I}^{2}$.

- T represents the total number of data points.

The process generating the trend can be regarded as a local approximation to a linear trend i.e.

$$
U_{t}=U_{t-1}+\beta_{t-1}+\eta_{t}
$$

Where $U_{t-1}$ is the stochastic level component and $\beta_{t-1}$ the slope parameter generated at the previous time step $t-1$. The component $\eta_{t}$ represents the disturbance term with zero mean and variance $\sigma_{\eta}^{2}$ [11].

The decomposition procedure is based on the locally-weighted regression (Loess) smoothing (STLplus) method of the R environment version 3.4.3 [12]. STL is an acronym for "Seasonal and Trend decomposition using Loess", a versatile and robust method for decomposing time series, and Loess is a method for estimating nonlinear relationships [13].

The algorithm works by running two loops:

- an outer loop where robustness coefficients are calculated to minimize the effect of outliers.

- an inner loop where the trend component and seasonal component are iteratively updated using Loess smoothing.

The algorithm can be set to run for a fixed number of iterations for each loop, or it can be set to run until a specific convergence criterion is met. The smoothing parameter of the seasonal component is an important parameter to define. It is represented by the frequency parameter which is the number of observations per cycle. For daily data, [13] recommends to use a window of at least 7 days (week). Missing values in the trend and seasonal components are handle by smoothing [12].

\section{Non-parametric statistical tests for trend estimation}

As specified in Fig. 2 two non-parametric statistical tests are performed on the datasets for trend estimation, i.e. the Mann-Kendall and Sen's slope tests.

\section{1) Mann-Kendall test}

The Mann-Kendall test analyzes differences between earlier and later data points. It is a rank-based and distribution-free test, widely adopted in time series analysis for detecting the presence of monotonic increasing or decreasing trends. The null hypothesis $(\mathrm{H} 0)$ of this non-parametric test states the absence of a monotonic trend, and the alternate hypothesis (H1) states the existence of a positive, negative, or non-null trend.
The principal statistics value $\mathrm{S}$ of the Mann-Kendall test is calculated using (3) - (5) [14].

$$
\begin{gathered}
S=\sum_{a-1}^{z-1} \sum_{b=a+1}^{z} \operatorname{sgn}\left(x_{b}-x_{a}\right) \\
\operatorname{sgn}\left(x_{b}-x_{a}\right)=\left\{\begin{array}{cc}
1 & \text { if } x_{b}-x_{a}>0 \\
0 & \text { if } x_{b}-x_{a}=0 \\
-1 & \text { if } x_{b}-x_{a}<0
\end{array}\right.
\end{gathered}
$$

Where $z$ is the total number of data points, and $x_{a}$ and $x_{b}$ are individual data points with $b$ greater than $a$.

$$
\begin{gathered}
\quad \operatorname{variance}(S)= \\
\frac{z(z-1)(2 z+5)-\sum_{t} t(t-1)(2 t+5)}{18}
\end{gathered}
$$

Here $t$ denotes the range of any assumed tie of sample points. $\sum_{t}$ represents the summation of all ties. The normal standard test statistics $\tau$ for estimating the presence of a statistically significant trend is calculated using (6) [15].

$$
\tau= \begin{cases}\frac{S-1}{\sqrt{\text { variance }(S)}} & \text { if } S>0 \\ 0 & \text { if } S=0 \\ \frac{S+1}{\sqrt{\text { variance }(S)}} & \text { if } S<0\end{cases}
$$

A negative or positive $\tau$ value corresponds to a decreasing or increasing trend, respectively, at the significance level $\alpha=0.05$ (i.e. at $95 \%$ of confidence level).

The Mann-Kendall test assumes that a value can always be declared less than, greater than, or equal to another value; that data are independent; and that the distribution of data remains constant in either the original units or transformed units [16]. In addition to not needing to conform to any particular distribution, this test is also resistant to outliers and missing values [17]. For these reasons, the method is applied without prior transformation of the data.

\section{2) Sen's slope test}

The Sen's slope test (or Theil-Sen estimator) is a widely used statistical test for non-parametric data to estimate the power of trend detected through the Mann-Kendall test [18]. Positive and negative values for the Sen's slope indicate upward and downward trends, respectively. Assuming a linear trend, the Sen's slope is the median of slopes

$$
Q=\frac{y_{j}-y_{k}}{t_{j}-t_{k}}, \quad j>k
$$

between data points $y_{j}$ and $y_{k}$ during time interval $t_{j}-t_{k}[14$, 19], corresponding to a span loss change per unit of time in our case. 


\section{Linear regression for trend validation}

Finally, after estimating the trend, we proceed to its validation, as mentioned in Fig. 2. To do this, the Theil-Sen method of linear regression, that chooses the median slope among all lines through pairs of two-dimensional sample points $\left(y_{j}, y_{k}\right)$ is used $[20,21]$. The equation is obtained as:

$$
y=\hat{\beta}_{0}+\hat{\beta}_{1} t
$$

Parameter $\hat{\beta}_{1}$ is the non-parametric slope estimate. It is the median of all $\mathrm{Q}$ values in (7) during the observation period. $\hat{\beta}_{0}$ is a constant called the intercept and $t$ is time.

$$
\hat{\beta}_{0}=y_{M E D}-\hat{\beta}_{1} \times t_{M E D}
$$

Where $y_{M E D}=$ median of $n$ data points $y_{1}, y_{2}, \ldots, y_{n}$

$t_{M E D}=$ median of $n$ time points $t_{1}, t_{2}, \ldots, t_{n}$

From (8) and (9), the estimated linear line $y$ can be rewritten as [22]:

$$
y=y_{M E D}+\hat{\beta}_{1}\left(t-t_{M E D}\right)
$$

\section{RESUlTS AND DISCUSSIONS}

The raw span loss data, as well as the results of the time series decomposition into three constitutive components (seasonal, trend, and remainder or noise) can be found for both directions in Fig. 6 and Fig. 9, for spans 1, 3 and, Fig. 7 and Fig. 10 , for spans 2, 4. First, we can see that the seasonal component (c) is very low $(<0.01 \mathrm{~dB})$. This is expected because it is a buried link. Thus, the seasonal component exists but it seems to indicate that temperature has no impact on the variability of the optical link, since it is not directly exposed to the elements of the changing seasons beyond temperature. We also note that the anomalies (or spikes) and noise in the raw span loss curves can be found in the remainder component (d), and that the resulting trend component is a smooth curve. The skewness of remainder component (d) is between -0.5 and 0.5 , which confirms that the remainder component follows the sigma squared distribution described earlier.

Apart from non-systematic patterns or events in the data, the remainder could provide some indication of the appropriate fit of the other component.

A high correlation in this component is an indication that some patterns related to other components were left over due to an inaccurate estimate [23] (see the remainder component plot (d) of span 2 for direction B to A, in Fig. 10).

The trend components for the 8 fiber spans are shown in Fig. 8 and Fig. 11. A slightly upward trend, barely visible in the raw data, can be seen slightly better in the trend component for fiber span 1 in direction A-B. This trend is also observed in the opposite direction as expected. It seems to reflect a degradation of the fiber, of which we will follow the evolution in the next part of the analysis. Several factors may influence the degradation, including, among others thing, age, environmental conditions and so on.
The trend component for fiber span 2 in both directions (Fig. 7 and 10, respectively) shows sudden changes in span loss, which can introduce some uncertainty on the calculated $\mathrm{Q}_{\text {median }}$ value. The trend extraction in this case captured these step-like events likely caused by fiber re-splicing or cable reconfiguration.

The trend components for fiber spans 3 - 4 do not show significant changes during the observation period. Despite the differences highlighted in each span, the statistical tests have been applied to the four spans.

The next step is to determine the slope of the trend component using the Mann-Kendall and Sen's slope statistical tests. We used the R library 'modifiedmk' to perform both tests at confidence level of 0.95 . The results are shown in TABLE II. Span 1 shows a change of $0.2 \mathrm{~dB} /$ year in one direction (A-B) and $0.08 \mathrm{~dB} /$ year in the opposite direction (B-A), with an average of $0.15 \mathrm{~dB} /$ year. Span 2 shows a change of 0.22 $\mathrm{dB} /$ year and $0.15 \mathrm{~dB} /$ year. The positive values for Kendall's $\tau$ parameter and S-value indicate the presence of a positive trend. The change percentage $(\mathrm{Pc})$ of the trend component is calculated by assuming a linear trend. That is, change percentage equals median slope (Sen's slope) multiplied by the period length (number of data points from which we are calculating the mean) divided by the corresponding mean [24]:

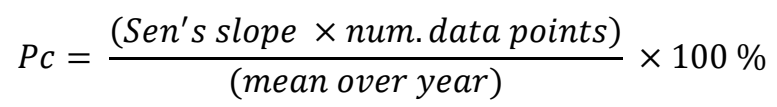

In order to validate the test results, i.e. to check the evolution in $\mathrm{dB} /$ year over the following year, the span 1 was selected as its loss shows a gradual positive trend. Fig. 12 shows the span loss data for the first 12 months (in black) and the additional 12 months (in blue) for both directions. The span loss increases in the first months of the second year, as expected, but seems to saturate at $13.7 \mathrm{~dB}$. The same saturation behavior is observed in the opposite direction. Independent span loss measurements obtained through the optical supervisory channel (OSC), although less accurate, confirmed that the observed trend was due to the fiber plant itself.

A linear line equation based on the first 12-month dataset (in black) was estimated and fitted over an additional 12-month dataset (in blue). The Theil-Sen, fitting Median - Based Linear Models (mblm) is built from the first twelve months. Fig. 13 shows the straight fitted line (in red) applied to the current span loss datasets (in black), and to the additional months (in blue on the plot). The intercept and slope of these lines are presented in TABLE III. The regression model is found to be significant at a $5 \%$ significance level with a low residual standard error (TABLE III). The prediction interval represented by the dotted line, is the region that contains approximately $95 \%$ of the measurements. This means that, if another measurement is taken, there is a $95 \%$ chance that it falls within the dotted prediction band. 

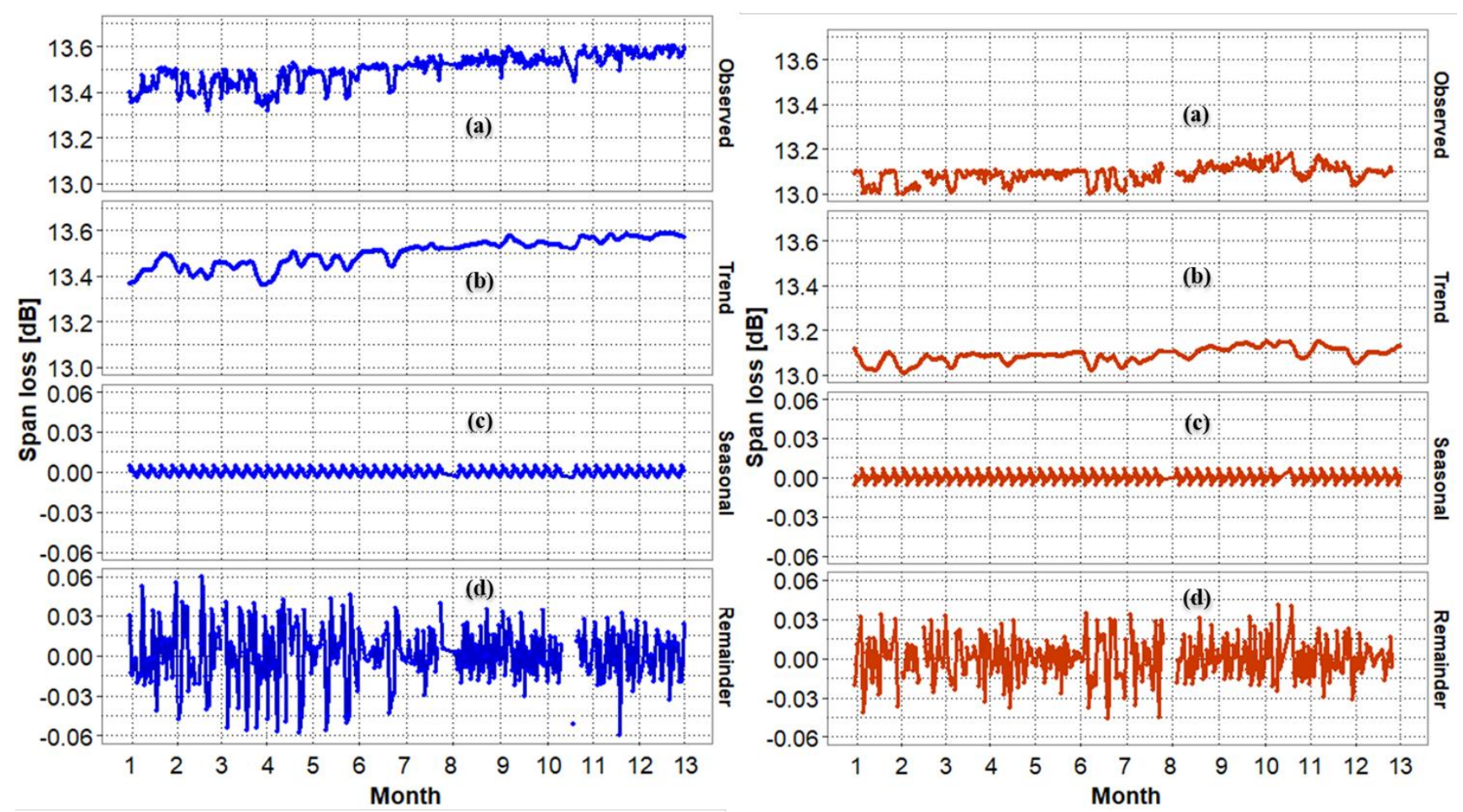

Fig. 6. Direction A - B : Time series decomposition plot for the 13-dB spans [span 1(blue), span 3(red)]: raw data (a); trend component (b); seasonal component (c); remainder (d)
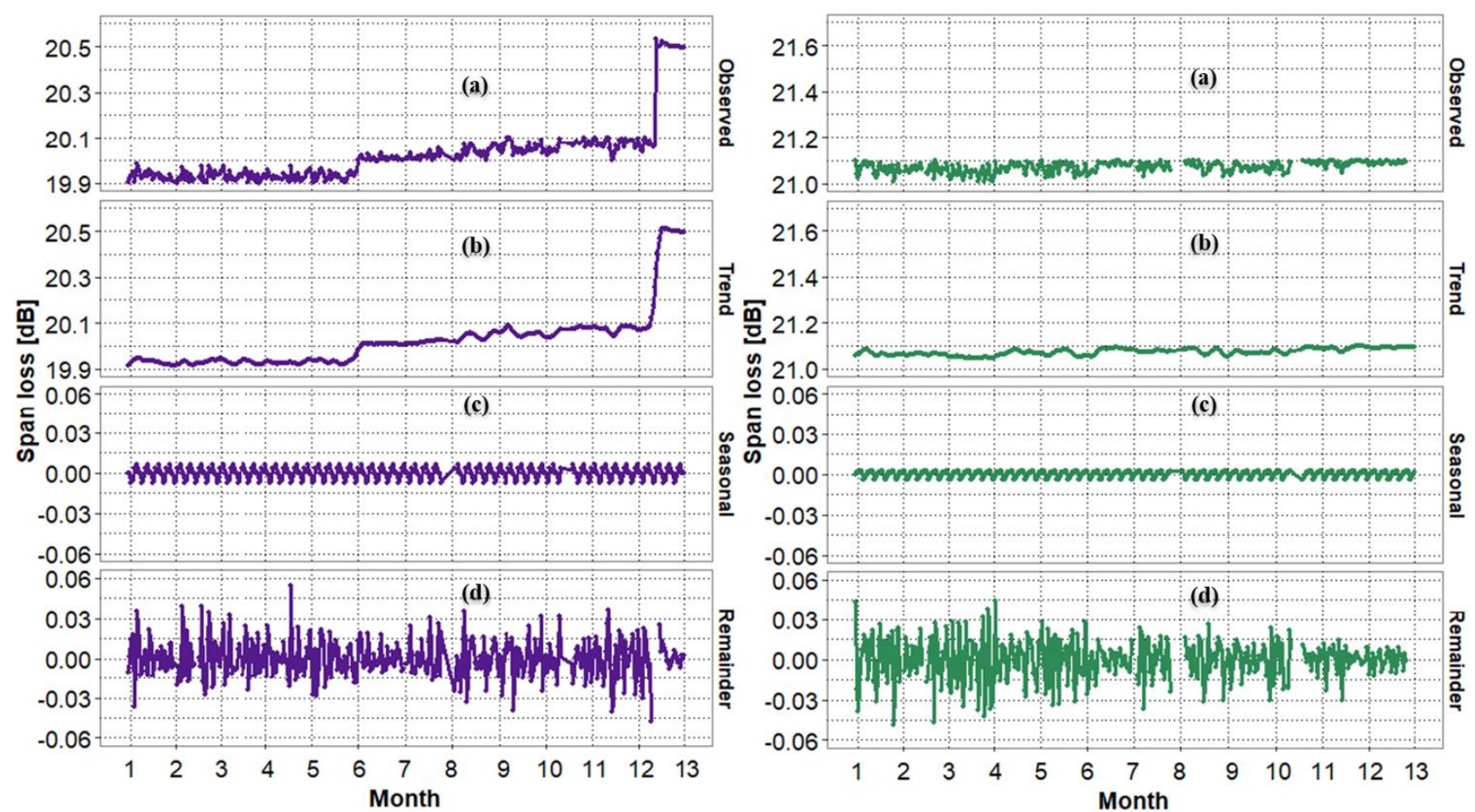

Fig. 7. Direction A - B : Time series decomposition plot for the 20-dB spans [span 2 (purple), span 4 (green)]: raw data (a); trend component (b); seasonal component (c); remainder (d) 

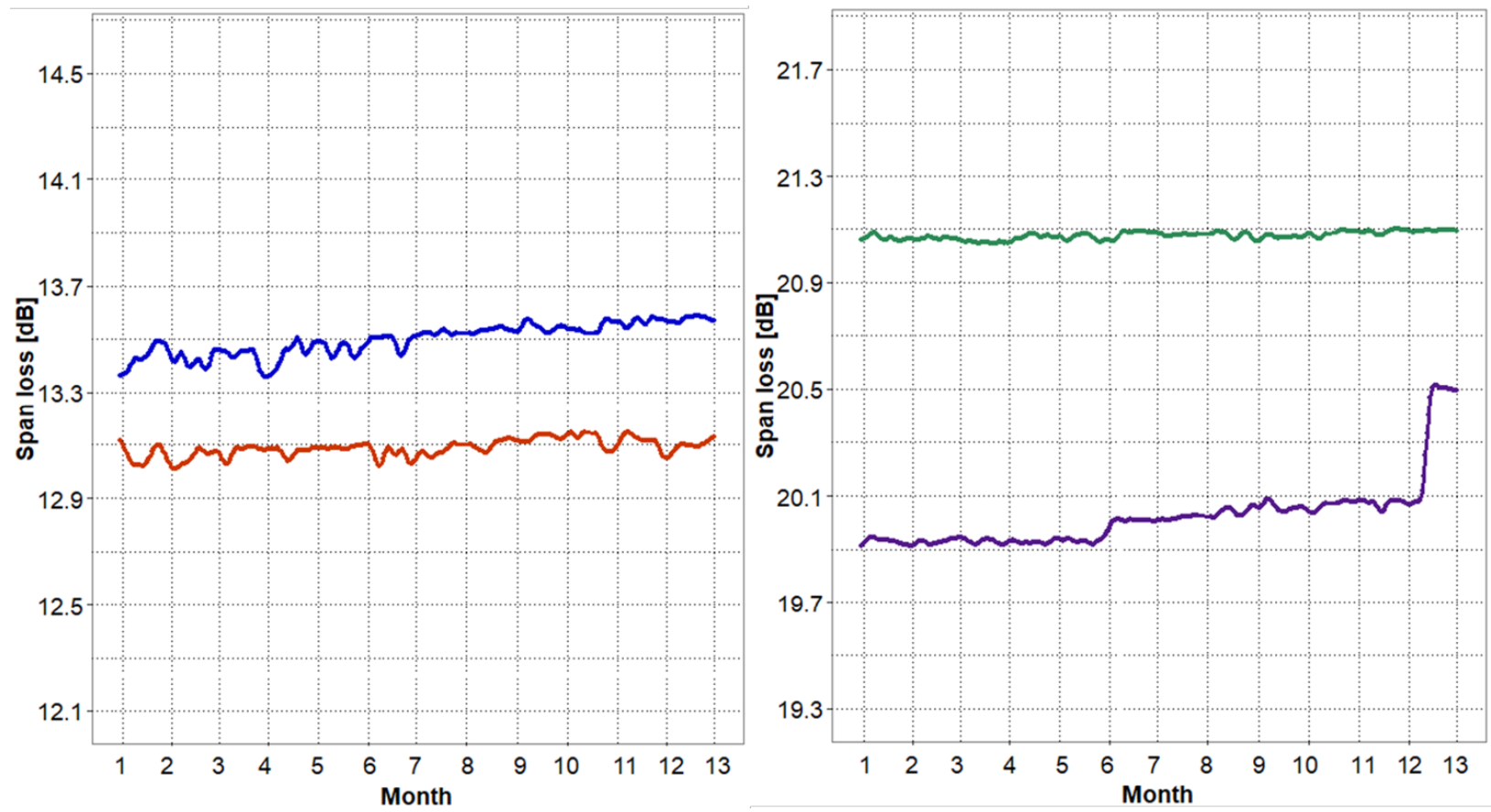

Fig. 8. Direction A - B : Trend components for span 1 (blue), span 3 (red), span 2 (purple), span 4 (green)
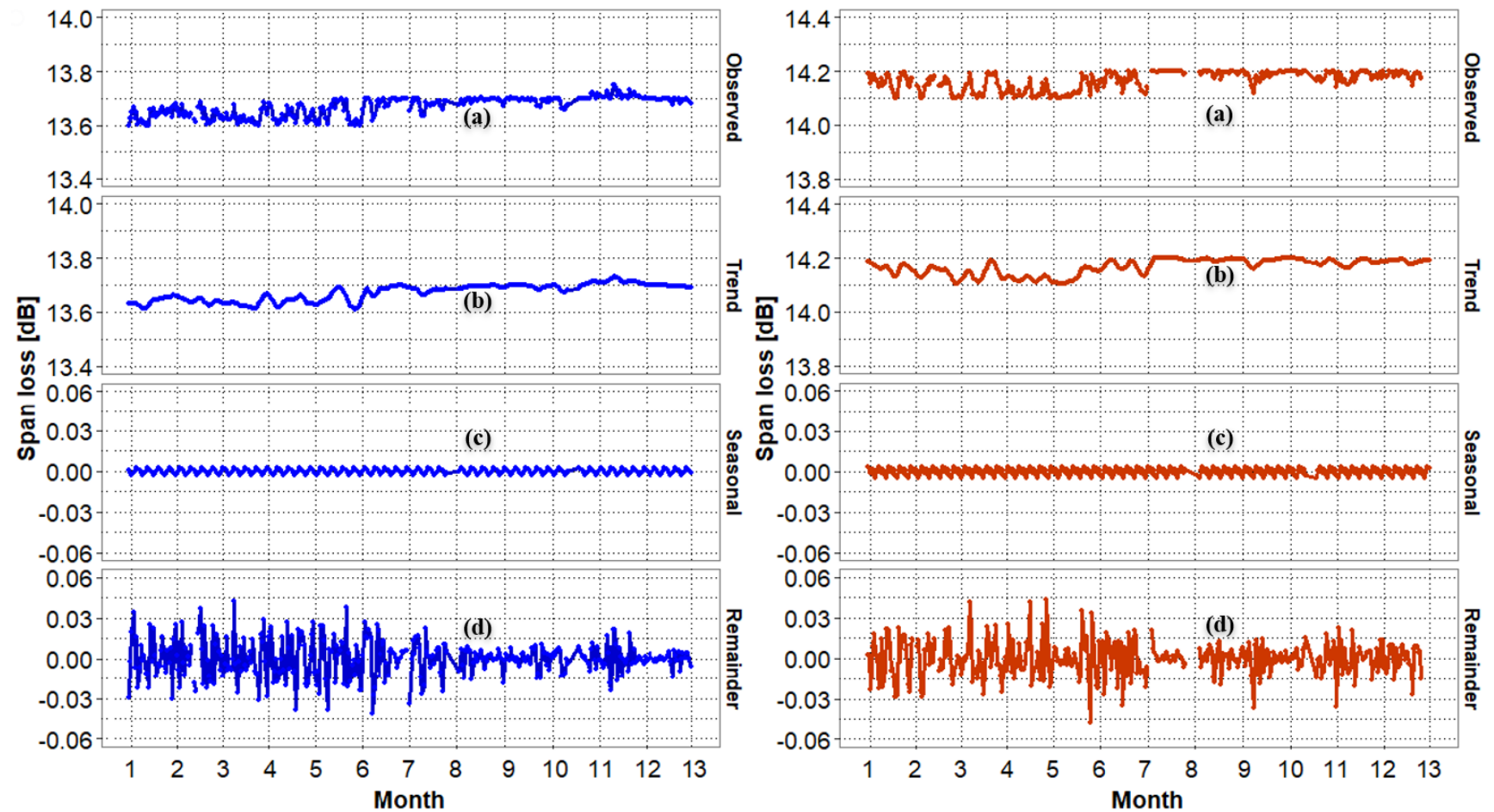

Fig. 9. Direction B - A : Time series decomposition plot for the 14-dB spans [1(blue) and 3(red)]: raw data (a); trend component

(b); seasonal component (c); remainder (d) 

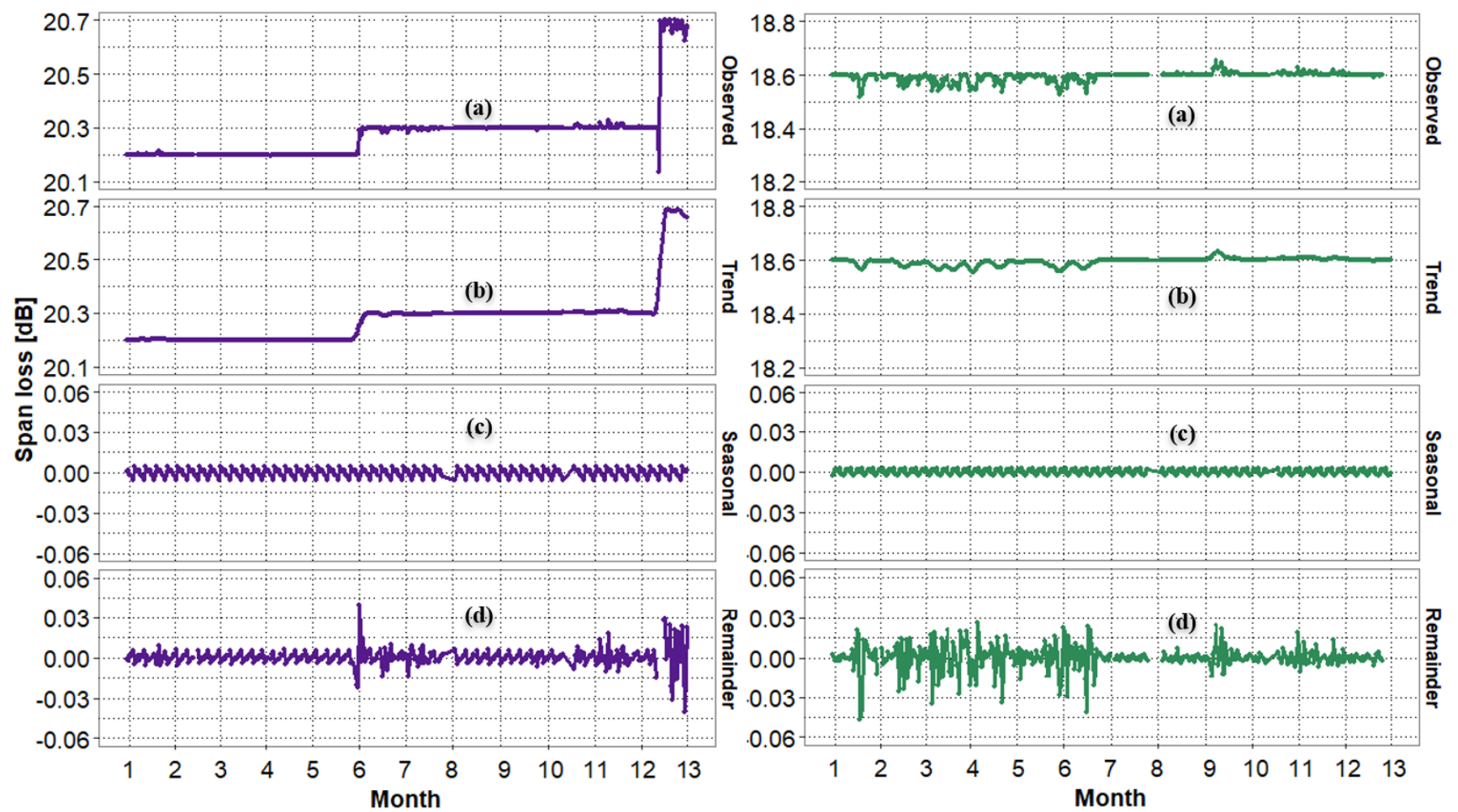

Fig. 10. Direction B - A : Time series decomposition plot for the $18-20-d B$ spans [2(purple) and 4(green)]: raw data (a); trend component (b); seasonal component (c); remainder (d)
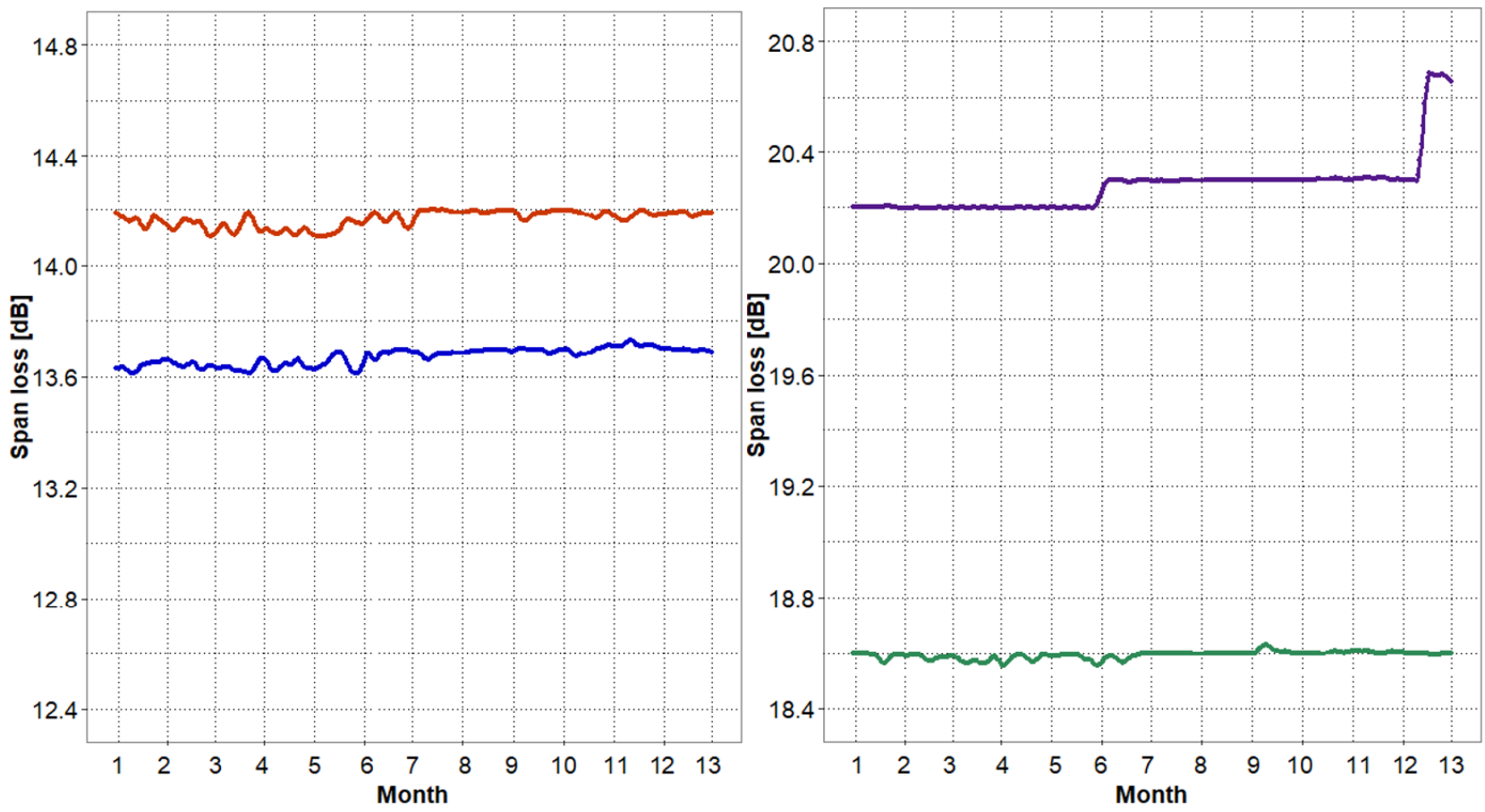

Fig. 11. Direction B - A : Trend components for span 1 (blue), span 3 (red), span 2 (purple), span 4 (green) 
TABLE II

STATISTICAL ANALYSIS OF THE SPAN LOSS DATA

\begin{tabular}{ccccccc}
\hline \hline & Span & $\tau$ & $\begin{array}{c}\text { S } \\
\left(\mathrm{x} 10^{4}\right)\end{array}$ & $\begin{array}{c}\text { Qmedian } \\
\left(\mathrm{x} 10^{-4}\right)\end{array}$ & $\begin{array}{c}\text { Change } \\
(\mathrm{dB} / \text { year })\end{array}$ & $\begin{array}{c}\text { Pc } \\
(\%)\end{array}$ \\
\hline \multirow{3}{*}{ Direction } & 1 & 0.7 & 4.7 & 5.0 & 0.20 & 1.3 \\
A-B & 2 & 0.7 & 4.6 & 6.2 & 0.22 & 1.1 \\
& 3 & 0.4 & 2.6 & 1.9 & 0.07 & 0.5 \\
& 4 & 0.4 & 2.9 & 0.9 & 0.03 & 0.2 \\
\hline & 1 & 0.6 & 4.0 & 2.3 & 0.08 & 0.6 \\
Direction & 2 & 0.7 & 4.3 & 4.2 & 0.15 & 0.7 \\
B-A & 3 & 0.4 & 2.3 & 1.5 & 0.05 & 0.4 \\
& 4 & 0.5 & 3.0 & 0.6 & 0.02 & 0.1 \\
\hline \hline
\end{tabular}

TABLE III

FITTED STRAIGHT LINES PARAMETERS AND RESIDUAL STANDARD ERROR (S)

\begin{tabular}{lccc}
\hline & Intercept (dB) & Slope $\left(10^{-4}\right)$ & $\boldsymbol{s}(\mathbf{d B})$ \\
\hline Span 1 (A-B) & 5.31 & 4.73 & 0.028 \\
Span 1 (B-A) & 9.47 & 2.43 & 0.019 \\
\hline \hline
\end{tabular}

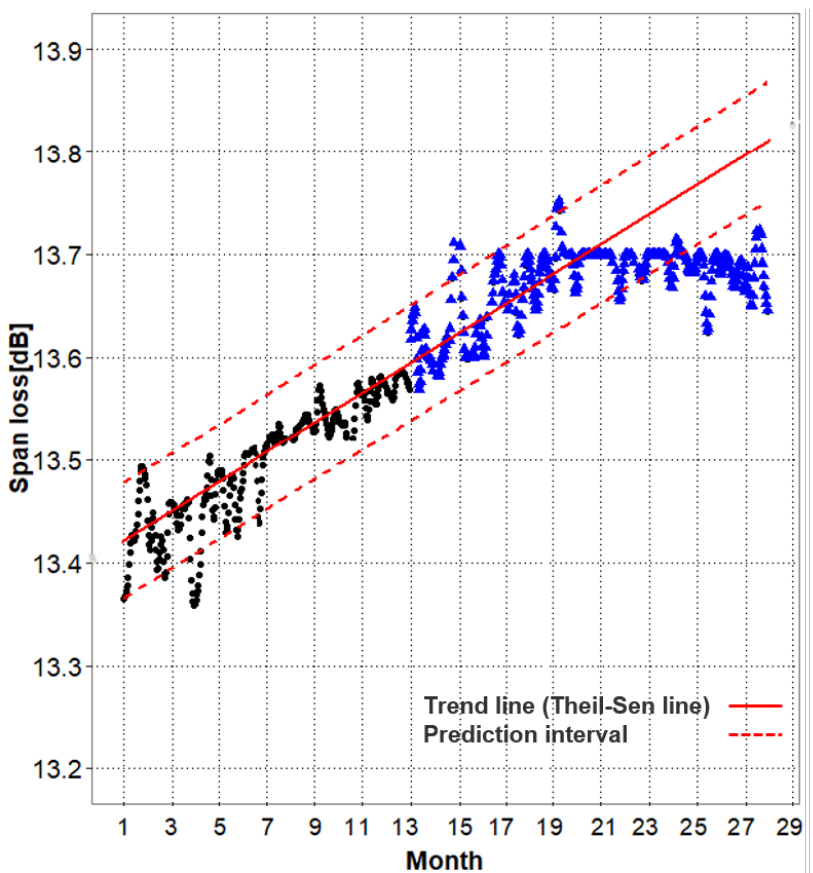

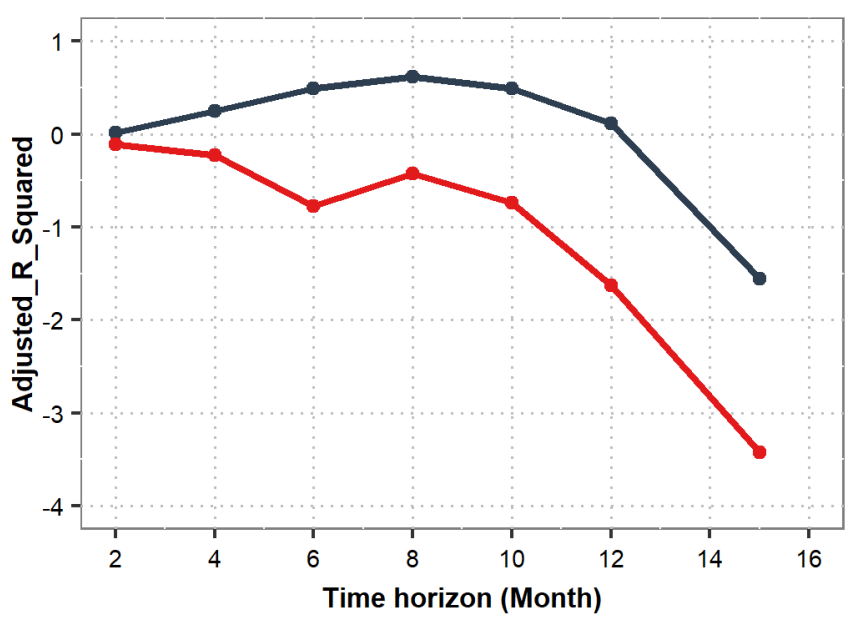

Fig. 12. Adjusted $\mathrm{R}^{2}$ curves as a function of the time horizon in the additional 12-month observation period : Direction A - B (black), Direction B - A (red)

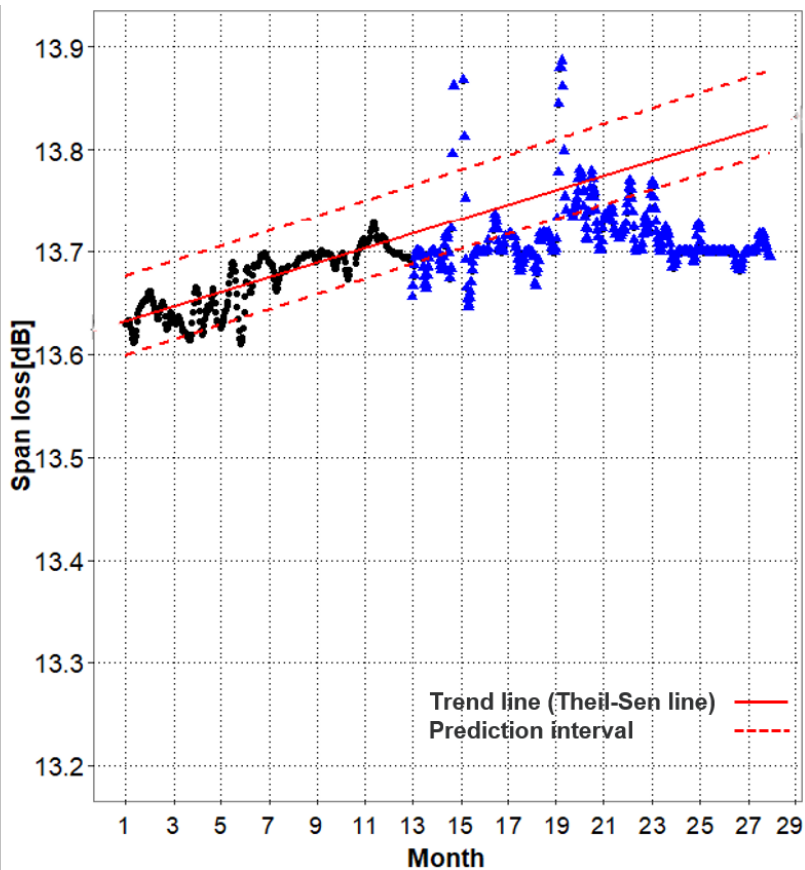

Fig. 13. Span loss data for Span 1: first 12-month observation period (in black), additional 12-month dataset (in blue) - (Direction A to B) and span 1 (Direction B to A)

The goodness of the regression fit is estimated by computing the adjusted $\mathrm{R}^{2}$ as [25]:

$$
R^{2}{ }_{a d j}=1-\frac{(n-1)}{(n-p)} \times \frac{S S E}{S S T}
$$

Where SSE is the sum of the squared errors, SST is the total sum of squares, $\mathrm{n}$ is the number of observations and $\mathrm{p}$ is the number of independent variables.

Fig. 12 shows the adjusted $\mathrm{R}^{2}$ curves as a function of the time horizon in the additional 12-month dataset (in blue).

In the direction $(\mathrm{A}-\mathrm{B})$, the $\mathrm{R}^{2}$ value increases for a time horizon up to 8 months, which validates the trends observed during the first 12 months of observation, and drops as the trend line levels off. In the direction (B - A), the adjusted $\mathrm{R}^{2}$ values are negative as the error exceeds the variance for all time horizons.

\section{CONCLUSION}

In this paper, the time series decomposition method has been applied to field span loss data collected in a $205-\mathrm{km}$ optically amplified link of a production network during a 12-month period, with the objective to detect potential long-term fiber loss degradation in the 8 fiber spans under study. The Mann-Kendall and the Sen's slope tests were used to estimate the span loss change. The results show a percentage change of $1.3 \pm 0.002 \%$ over the 12 -month observation period for fiber span 1 or about $0.2 \mathrm{~dB}$. The measured span loss increase 
detected in this case study is very small, as expected over relatively short observation period. The detected increase in span loss for span 1 was validated by applying a linear regression over an additional 12-month dataset, which confirmed the trend and a $0.3-\mathrm{dB}$ increase in span loss over a 19-month period (direction A to B). A saturation of the span loss at $13.7 \mathrm{~dB}$ was observed in both directions. As confirmed by independent loss measurements through OSC, the observed trend and saturation of the span loss at $13.7 \mathrm{~dB}$ was due to the fiber plant itself, and not to measurement or instrumentation issues. Further investigation using additional span loss data over longer observation periods will be required to evaluate the prediction accuracy of our proposed method and to get a better understanding of the span loss dynamics in installed optical fiber cables.

The objective of this study was to show how the time series decomposition method combined with statistical analysis could be used to detect long-term performance trends in optical networks [26]. The method has been used for the analysis of historical fiber span loss data collected in a production network over an observation period of several months. Such span loss data is readily available for the network operators and does not require the use of additional instrumentation such as OTDRs. This tool would be useful for analysis of span loss data, including identifying where "unpredictable" events (or breakpoints) and changes in trend have occurred. If automated, this method could be used by network operators for fiber surveillance aiming at early detection of fiber plant degradation and proactive planning of fiber replacement using existing collected data from legacy installations. The method would also be useful for quantifying the seasonality component in fiber span loss or other performance metrics such as BER. Finally, the method could be applied to identify and locate the unpredictable events from the raw observations for further analysis of the remainder (noise) component.

\section{REFERENCES}

[1] T. Xu et al., "Field trial over $820 \mathrm{~km}$ installed SSMF and its potential Terabit/s superchannel application with up to 57.5-Gbaud DP-QPSK transmission," Optics Communications, vol. 353, pp. 133-138, 2015/10/15/2015.

[2] A. E. Willner, Z. Pan, and C. Yu, Optical performance monitoring. Elsevier Inc, 2008.

[3] M. Poulain and I. Severin, Aging and reliability of single-mode silica optical fibers in Optical Fibers Research Advances. 2007.

[4] J. Matthewson, "Optical fiber reliability models," in SPIE Critical Reviews of Optical Science and Technology, New Jersey, 1993.

[5] "Fatigue testing of silica optical fibres," Journal of Optoelectronics and Advanced Materials, pp. 1581-1588, 2005.

[6] R. Gazdzinski, "Cable and method of monitoring cable aging," San Diego, California Patent 5,902,962, 1999.

[7] A. Maslo, M. Hodzic, E. Skaljo, and A. Mujcic, "Aging and Degradation of Optical Fiber Parameters in a 16-Year-Long Period of Usage," Fiber and Integrated Optics, vol. 39, p. 39, January 1, 2020.

[8] F. Aguilera, F. Orlandi, L. Ruiz-Valenzuela, M. Msallem, and M. Fornaciari, "Analysis and interpretation of long temporal trends in cumulative temperatures and olive reproductive features using a seasonal trend decomposition procedure," Agricultural and Forest Meteorology, vol. 203, pp. 208-216, 2015/04/15/2015.

[9] R. Ali, A. Kuriqi, S. Abubaker, and O. Kisi, "Long-Term Trends and Seasonality Detection of the Observed Flow inYangtze River Using
Mann-Kendall and Sen's Innovative Trend Method," Water, 5 septembre 2019.

[10] K. W. Hipel and A. I. McLeod, "Chapter 23 Nonparametric Tests for Trend Detection," in Developments in Water Science, vol. 45, K. W. Hipel and A. I. McLeod, Eds.: Elsevier, 1994, pp. 853-938.

[11] A. C. Harvey and S. Peters, "Estimation procedures for structural time series models," Journal of Forecasting, vol. 9, no. 2, pp. 89-108, 1990.

[12] R. Hafen. (2016). Enhanced Seasonal Decomposition of Time Series by Loess. https://cran.r-project.org/web/packages/stlplus/stlplus.pdf

[13] R. B. Cleveland, W. S. Cleveland, J. E. McRae, and a. I. Terpenning, "STL: A Seasonal-Trend Decomposition Procedure Based on Loess " Journal of Official Statistics, vol. Vol. 6. No. I. , pp. pp. 3-73, 1990.

[14] A. Balasubramanian, K. Duraisamy, S. T, S. Krishnaraj, and M. Suresh, "Long-term trend detection and spatiotemporal analysis of groundwater levels using GIS techniques in Lower Bhavani River basin, Tamil Nadu, India," Environment, Development and Sustainability, 01/21 2019.

[15] A. Jaiswal, C. Samuel, and V. M. Kadabgaon, "Statistical trend analysis and forecast modeling of air pollutants," Global Journal of Environmental Science and Management, vol. 4, pp. 427-438, 11/14 2018.

[16] D. R. Helsel and R. M. Hirsch, S. U.S. Department of the interior gale a. Norton, Ed. Statistical Methods in Water Resources http://water.usgs.gov/pubs/twri/twri4a3/ 2002, p. 527.

[17] D. W. Meals, J. Spooner, S. A. Dressing, and J. B. Harcum. (2011, 2020-04-21). Statistical Analysis for Monotonic Trends. Available: https://www.epa.gov/sites/production/files/2016-05/documents/tech_no tes 6 dec 2013 trend.pdf

[18] T. Caloiero, R. Coscarelli, E. Ferrari, and B. Sirangelo, "Trend analysis of monthly mean values and extreme indices of daily temperature in a region of southern Italy," International Journal of Climatology, vol. 37, no. S1, pp. 284-297, 2017.

[19] O. Olofintoye, J. Adeyemo, and F. Otieno, "Impact of Regional Climate Change on Freshwater Resources and Operation of the Vanderkloof Dam System in South Africa " in Global warming, impacts and future perspective B. R. Singh, Ed. Croatia: Janeza Trdine 9, 51000 Rijeka 2012, p. 364.

[20] D. Helsel and R. Hirsch, Statistical Methods in Water Resources. 1992.

[21] W. J. Conover, Practical nonparametric statistics. Wiley, 1999.

[22] L. P. N. National. (09 September ). Nonparametric Estimate of Trend. Available: https://vsp.pnnl.gov/help/index.htm\#Vsample/Nonparametric Estimate of Trend

[23] R. Krispin, Hands-On Time Series Analysis with R: Perform time series analysis and forecasting using $R$. Birmingham: Packt Publishing Ltd., 2019, p. 418.

[24] A. K. Taxak, A. R. Murumkar, and D. S. Arya, "Long term spatial and temporal rainfall trends and homogeneity analysis in Wainganga basin, Central India," Weather and Climate Extremes, vol. 4, pp. 50-61, 2014/08/01/2014.

[25] G. Grekousis, Spatial Analysis Theory and Practice: Describe - Explore - Explain through GIS. United Kingdom: Cambridge University Press, 2020.

[26] Q. Wen, J. Gao, X. Song, L. Sun, H. Xu, and S. Zhu, "RobustSTL: A Robust Seasonal-Trend Decomposition Algorithm for Long Time Series," Association for the Advancement of Artificial Intelligence p. 9, 2018.

Banti Laure M. Yaméogo received the B.Eng. degree in System and Network Management from the University Aube Nouvelle, Burkina Faso, in 2005 and the M.Ing. degree from the École de technologie supérieure, Montréal, Canada in 2015. Her Master's project focused on the development of a simulation model for a $100 \mathrm{~Gb} / \mathrm{s}$ coherent optical link. Since 2016, she is working towards the Ph.D. degree at École de technologie supérieure, with the Network Technology Lab. Her research interests include statistical analysis and characterization of performance monitoring datasets collected in optical networks. 
Doug Charlton received the B.Eng. degree in Electrical Engineering from Carleton University, Ottawa, Canada, in 2012. He works at Ciena Corp. since 2012 where he contributes to testing and automation as part of the modem development.

David Doucet holds B.Eng with distinction (1983) and M.Eng (1986) degrees in Electrical Engineering from Carleton University. He is currently Director of Modem Systems Development at Ciena. He has held various positions in industry and has spent the last 32 years bringing many optical telecommunications products to market, spanning single wavelength traffic rates from $2.5 \mathrm{~Gb} / \mathrm{s}$ up to $800 \mathrm{~Gb} / \mathrm{s}$, initially working in the development of ASICs, and subsequently managing all aspects of hardware development (ASIC, FPGA, PCB, DSP, FW, optics, shelf infrastructure, and ultimately, systems). For the past 12 years, he has headed up a modem systems development team, defining requirements for, and validating capabilities of, coherent optical modem products, beginning with the world's first coherent product, WaveLogic 2, and all subsequent WaveLogic technology families, including the latest, WaveLogic 5, $800 \mathrm{~Gb} / \mathrm{s}$ capable product.

Christian Desrosiers received a Ph.D. degree in applied mathematics from École Polytechnique de Montréal, Montreal, Canada, in 2008, He is working, since 2009, as assistant professor in the Software and IT Engineering department at ÉTS. Before joining the department, he was a postdoctoral research assistant at the University of Minnesota, USA. His current research activities focus on the development and applications of machine learning techniques to solve problems in the fields of data mining, computer vision, biomedical engineering, recommender systems, social network analysis, business intelligence and optical networking.

Maurice O'Sullivan has engineered the physical layer of optical transmission for more than 30 years, at first in the optical cable business, developing factory-tailored metrology for optical fiber, but, mainly, in the optical transmission business developing, modeling and verifying physical layer designs and performance of Nortel's (now Ciena's) line and highest rate transmission product including the first commercial $10 \mathrm{~Gb} / \mathrm{s}$ system, several commercial terrestrial line systems, the first commercial DSP assisted electric field modulation transceiver with complete electronic compensation for optical dispersion and the first commercial coherent $40 \mathrm{~Gb} / \mathrm{s}$ and $100 \mathrm{~Gb} / \mathrm{s}$ transceivers. Now with Ciena, Maurice is engaged in the design of the next generation of flexible high capacity coherent transceivers following on the recent commercial success of Ciena's multi-rate $50 \mathrm{G} / 100 \mathrm{G} / 200 \mathrm{G}$ product. Maurice is a Ciena Fellow with more than 45 patents and holds a Ph.D. in physics (high resolution spectroscopy) from the University of Toronto.

Christine Tremblay received the B.Sc. degree in Engineering Physics from Université Laval, Quebec City, Canada, in 1984, the M.Sc. degree (Energy) from INRS-Énergie, Varennes, Canada, in 1985 and the Ph.D. degree (Optoelectronics) from the École Polytechnique de Montréal, Canada, in 1992. She is a Full Professor with the Department of Electrical Engineering and Associate Director for the Ph.D. Program at the École de technologie supérieure. She is the Founding Researcher and Head of the Network Technology Lab. Before joining the ÉTS, she was a Research Scientist with the National Optics Institute (INO) where she conducted research on integrated optical devices for communication and sensing applications. She held senior R\&D and technology management positions for several organizations. As Engineering Manager at EXFO and Director of Engineering at Roctest, she was responsible for the development of fiber-optic test equipment. She also served as Product Manager at Nortel for DWDM systems. Her team pioneered the research on filterless optical networking. Her current research interests include machine learning for optical networking applications, as well optical performance monitoring and advanced access networks for $5 \mathrm{G}$ applications. She has been co-instructor for SC314 and SC210 hands-on courses of the Optical Society of America on optical fiber and polarization measurements (2009-2015).

Dr. Tremblay is a Senior member of IEEE and a member of the Optical Society of America (OSA) and STARaCom and COPL Strategic Clusters of FRQNT. She is currently serving as Program Chair for the Photonics Networks and Devices (NETWORKS) 2020 OSA Topical Meeting and Program Committee Member for the OFC 2021 Subcommittee N3: Architecture and software-defined control for metro and core networks. 